UCRL-ID-130429

\title{
The Spheromak Path to Fusion Energy
}

\author{
E.B. Hooper, C.W. Barnes, P.M. Bellan, M.R. Brown, J.C. Fernandez, \\ T.K. Fowler, D.N. Hill, T.R. Jarboe, L.L. LoDestro, H.S. McLean, \\ M.J. Schaffer, K.I. Thomassen, M. Yamada
}

April 1998

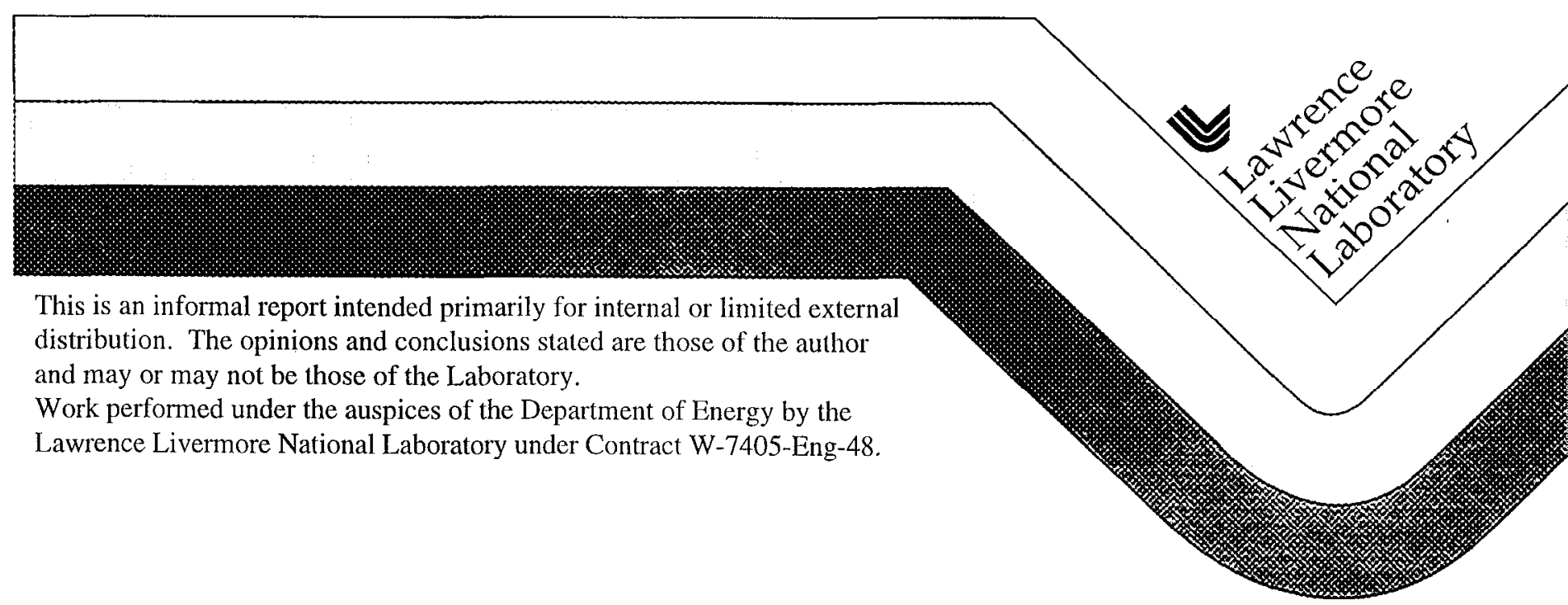




\section{DISCLAIMER}

This document was prepared as an account of work sponsored by an agency of the United States Government Neither the United States Government nor the University of California nor any of their employees, makes any watranty, express or implied, or assumes any legal liability or responsibility for the accuracy, completeness, or usefulness of any information, apparatus, product, or process disclosed, or represents that its use would not infringe privately owned rights Reference herein to any specific commercial product, process, or service by trade name, trademark, manufacturer, or otherwise, does not necessatily constitute or imply its endorsement, tecommendation, or favoring by the United States Government or the University of California The views and opinions of authors expressed herein do not necessarily state or reflect those of the United States Government or the University of California, and shall not be used for advertising or product endorsement purposes

This report has been reproduced directly from the best available copy

Available to DOE and DOE contractots from the Office of Scientific and Technical Information P O Box 62, Oak Ridge, TN 37831

Prices available from (615) 576-8401, FTS 626-8401

Available to the public from the

National Technical Information Service

U S Department of Commerce

5285 Port Royal Rd.

Springfield, VA 22161 


\title{
The Spheromak Path to Fusion Energy
}

\author{
April, 1998
}

This document is a community white paper describing the development path for the spheromak. It was prepared by an informal group of authors from the Magnetic Fusion Energy Community, including:

E. B. Hooper, editor (LLNL), C. W. Barnes (LANL), P. M. Bellan (Caltech), M. R. Brown (Swarthmore), J. C. Fernández (LANL), T. K. Fowler (U. California at Berkeley), D. N. Hill (LLNL), T. R. Jarboe (U. Washingtor), L. L. LoDestro (LLNL), H. S. McLean (U. California at Davis), M. J. Schaffer (General Atomics), K. I. Thomassen (LLNL), and M. Yamada (PPPL). 


\title{
The Spheromak Path to Fusion Energy
}

\author{
April, 1998
}

\section{Executive Summary}

The spheromak is a simple and robust magnetofluid configuration with several attractive reactor attributes including compact geometry, no material center post, high engineering $\beta$, and sustained steady state operation through helicity injection. Spheromak physics was extensively studied in the US program and abroad (especially Japan) in the 1980's with work continuing into the 1990s in Japan and the UK. Scientific results included demonstration of self-organization at constant helicity, control of the tilt and shift modes by shaped flux conservers, elucidation of the role of magnetic reconnection in the magnetic dynamo, and sustainment of a spheromak by helicity injection.

Several groups attained electron temperatures above $100 \mathrm{eV}$ in decaying plasmas, with CTX reaching $400 \mathrm{eV}$. This experiment had high magnetic field $(>1 \mathrm{~T}$ on the edge and $\sim 3 \mathrm{~T}$ near the symmetry axis) and good confinement. More recently, analysis of CTX found the energy confinement in the plasma core to be consistent with Rechester-Rosenbluth transport in a fluctuating magnetic field, potentially scaling to good confinement at higher electron temperatures. The SPHEX group developed an understanding of the dynamo in sustained spheromaks but in a relatively cold device. These and other physics results provide a foundation for a new "concept exploration" experiment to study the physics of a hot, sustained spheromak. If successful, this work leads to a next generation, proof-of-principle program.

The new SSPX experiment will address the physics of a large-scale sustained spheromak in a national laboratory (LLNL) setting. The key issue in near term spheromak research will be to explore the possibly deleterious effects of sustainment on confinement. Other important issues include exploring the $\beta$ scaling of confinement, scaling with Lundquist number $S$, and determining the need for active current-profile control. Collaborators from universities and other national laboratories are contributing experience from previous work, diagnostics, and physics support. Experiments at PPPL and Swarthmore are being conducted on the physics of magnetic reconnection, yielding physics results which should help advance the confinement work.

A spheromak reactor will require steady state operation with the equilibrium fully supported by external coils. Although the present generation of experiments can provide data on the initial stages of the Iransition from short-pulsed operation, sustainment longer than the wall resistance time will be addressed in the proof-of-principle experiments. 


\section{Contents}

I. Executive Summary 1

II. Vision 3

III. Basic Plasma Science and the Spheromak Path 3 towards a Low-Cost Fusion Reactor

IV. Development Path

A. Fusion confinement experiments 4

$\begin{array}{ll}\text { B. Theory and computations } & 7\end{array}$

C. Applications of spheromak research other than 9 magnetic confinement

V. Physics Issues and Goals 9

A. Scientific issues and goals 9

B. Technology issues and goals $\quad 12$

$\begin{array}{ll}\text { C. Energy issues and goals } & 13\end{array}$

VI. Existing Spheromak Programs and Resources 14

A. Confinement and helicity injection experiments 14

B. Physics of reconnection 16

C. CT injection for fueling 16

$\begin{array}{ll}\text { D. Diagnostic development } & 17\end{array}$

VII. Collaborations and Contributions to the White 17 Paper

$\begin{array}{ll}\text { References (main text) } & 18\end{array}$

$\begin{array}{ll}\text { Appendix Physics Issues and Status } & 20\end{array}$

$\begin{array}{ll}1 . & \text { Spheromak physics }\end{array}$

2. Recent results from the SPHEX experiment 23

References (appendix) 24 


\section{Vision}

Significant contributions to plasma and fusion science while advancing the spheromak, with its inherent compactness and its simple wall and magnet geometry, along the path to a reactor.

\section{Basic Plasma Science and the Spheromak Path towards a Low- Cost Fusion Reactor}

The spheromak involves a broad range of plasma science. Included are physics important to space plasmas such as the solar corona and the earth's magnetotail. Understanding and knowledge flow in both directions, enriching science in both space and laboratory, and it is likely that this synergy will continue.

The spheromak is a prime example of the role that helicity, a measure of linked magnetic fluxes, can play in determining the characteristics of hot plasmas. The important concept of helicity as a constraint (but not the name) was originated by Woltjer [1] in the astrophysical context; he showed that helicity was constant in a closed system, and: "Thus, the force-free fields with constant $\alpha\left[=j_{\|} / B\right]$ represent the lowest state of magnetic energy which a closed system may attain." The concept was first used on what we now call compact toroids by Wells and co-workers [2].

The concept was developed further by Taylor [3] and others for laboratory plasmas (RFPs, spheromaks) and space plasmas. Taylor's model, whereby energy decays subject to conservation of global magnetic helicity, provided a very simple, elegant description of how laboratory plasmas self-organize in the presence of strong MHD turbulence. Experiments and theory have demonstrated the validity of global helicity conservation and have advanced associated physics, including the role of magnetic fluctuations and reconnection in current drive via the magnetic dynamo.

The spheromak [4] has a robust and simple structure. Due in large measure to the helicity constraint, almost any initial configuration with sufficient helicity and energy will spontaneously relax to form a spheromak given appropriate boundary conditions. The simplicity and compact size of spheromaks provide excellent access for diagnostics and make spheromaks relatively inexpensive to build.

The golden age - to date - of US spheromak research was the decade 1980-90. US experiments included Beta-II and RACE at LLNL, PS at U. Maryland, CTX at LANL, and S-1 at PPPL; because of funding cutbacks even 
the best performers of these experiments were shut down by the early 1990's. Japanese experimental facilities were CTCC at Osaka, FACT at HIT and TS-3 at Tokyo. The Japanese experiments were typically smaller, but have continued operating although recently not as spheromaks.

Spheromaks have been formed with toroidal current $>1 \mathrm{MA}$ and surface magnetic fields $>1$ tesla. CTX demonstrated good enough confinement in a decaying plasma to achieve $T_{e}=400 \mathrm{eV}$ [5] and peak electron betas $>20 \%$. [6] Sustainment by electrostatic helicity injection was demonstrated in CTX and in SPHEX at the U. Manchester Institute of Science and Technology in England. (SPHEX has recently been shut down.) Results from the large number of previous spheromak experiments, found in several review articles $[7,8,9,10]$ and the appendix to this white paper, provide the basis for the new efforts.

The spheromak is potentially an attractive fusion reactor. It has no material central post. It has a simple wall and magnet geometry and potentially high engineering beta (the ratio of plasma pressure to magnetic field at the coils). It thus offers the possibility of a reactor with qualitative advantages over those requiring a toroidal first wall [11].

Electrostatic helicity injection can provide efficient current drive in a steady-state mode of operation for a reactor. The predominant scientific issues are whether good energy confinement can be obtained during sustainment by helicity injection, and whether the energy confinement time increases rapidly enough with temperature that a compact, high beta reactor is possible.

Pulsed reactors which operate on a time scale short compared with magnetic diffusion in the surrounding walls might avoid the issue of sustainment. The spheromak is a candidate for very high beta operation in a Magnetized Target Fusion (MTF) device. Alternatively, it may be possible to operate in an intermediate pulsed regime, to be achieved by rapid fueling after ignition is achieved at low beta.

\section{Development Path}

\section{A. Fusion confinement experiments}

The plasma current in a spheromak is sustained efficiently by the injection of helicity from an external source. Helicity current drive arises when 3-dimensional magnetic and fluid velocity fluctuations generate a "dynamo electric field," which balances resistive loses. Global MHD analysis [12] predicts the dynamo mechanism to be strong enough, relative to resistive losses, that it can function at very small magnetic fluctuation levels and thus 
with good energy confinement for reactor conditions. While there is overwhelming experimental evidence for the existence and effectiveness of the dynamo, the detailed physics of the required fluctuations and the scaling of their amplitude with the Lundquist number, $S$ (ratio of resistive time, $\tau_{R}$, to Alfvén time, $\left.\tau_{A}\right)$, are poorly understood.

Spheromaks involve a balance between two opposing requirements: on the one hand optimum confinement requires axisymmetry, but on the other hand sustaining this current via helicity injection is fundamentally a nonaxisymmetric process. Thus, the essential question is whether adequate confining current can be sustained with only a small amount of confinementdegrading non-axisymmetric modes. If the answer is positive, then a modest amount of confinement will be traded away for the engineering simplicity of helicity injection.

Theoretical models predict that pressure-driven "resistive-g" modes will be unstable, although finite Larmor radius (FLR), neoclassical, and fluid flow effects, among others, are not generally included. For the spheromak concept to succeed it is also important that these modes be stable or saturate at small island widths at high temperatures.

To determine the scalings and confinement and to address the other physics issues important to the spheromak, we anticipate a series of experimental facilities. ${ }^{*}$ These experiments require associated theoretical and computational support. The spheromak development path is envisaged to involve three stages:

- Concept Fxploration: Near-term confinement experiments (Section VI) are designed to evaluate energy confinement of sustained spheromaks and to study how magnetic fluctuations relate to helicity injection and affect confinement. These will advance spheromak physics and determine whether moderate electron temperatures $(100 \mathrm{~s} \mathrm{of} \mathrm{eV)} \mathrm{can} \mathrm{be}$ achieved during sustainment of the plasma for moderate times (a few $\mathrm{ms}$ ). In these experiments, transient wall image currents will provide both the axisymmetric equilibrium vertical field and the shaping to prevent tilt and shift instabilities. The size and magnetic fields of the experiments must yield a sufficiently large $S$ that the physics on the Alfvén time can be separated from that on resistive times. Further, the reconnection time, $\sim S^{-3 / 5} \tau_{R}$, must be short compared to internal physics times. For reconnection to dominate over resistive diffusion, $S>10^{4}$ is required $[10,13]$.

${ }^{*}$ The development stages addressed here are from the Alternate Concepts Panel report to the Fusion Energy Sciences Advisory Board, July 1996,

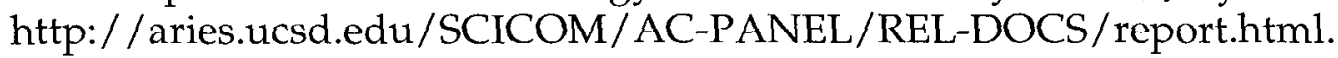


- Proof-of-Principle: These experiments will involve long-pulse operation for which wall image currents will be inadequate to provide equilibrium and stability. The required equilibrium fields will be produced by external coils, allowing long duration compared to the $\mathrm{L} / \mathrm{R}$ time of the conducting wall. It also secms likcly that feedback control of resistive wall modes will be required. (The tilt and shift modes become resistive wall modes in the presence of a finite resistivity flux conserver.) Physics addressed in these experiments include confinement and other properties in the steady-state plasma. These experiments will demonstrate "proof-ofprinciple."

- Proof of Performance and Optimization: If successful, the proof-ofprinciple experiments will be followed by a proof-of-performance and optimization experiment for the spheromak. Although it is premature to characterize this experiment in detail, it would operate at or near the fusion regime, but without a burning plasma. Success at this stage would lead to the fusion energy development and demonstration stages. It would operate for durations comparable to wall recycling times (but not wall equilibration times), with $\mathrm{keV}$ tempcratures in the core.

Theory and modeling to support these experiments are described in the next section. Detailed issues and goals for the program are described in Section IV.

Collaborations both within the spheromak community and with other approaches to fusion are essential as this program proceeds, and will require appropriate resources. (The present status of collaborations is described in Section VII.) As the program proceeds to larger and more expensive experiments, it will become increasingly "national" in scope.

Because spheromaks are related to other alternate concepts, much can be learned from their physics. Among related approaches, the closest is the RFP. It is based on similar physics, but it is made in a toroidal chamber with applied toroidal magnetic flux and has a transformer as the primary current drive. There are important differences, e.g. in the RFP, helicity is generated by injection of poloidal flux, for example by ohmic drive; and the spheromak, as presently envisioned on the reactor path, is driven by electrostatic helicity injection through a coaxial plasma source injecting toroidal flux. The safetyfactor $(q)$ profiles are very different, with the RFP having a lower $q$ but with more magnetic shear due to field reversal. The dominant RFP modes have $m=1$ and are thus global ("non-constant $\psi$ ") in nature. As a result of its higher $q$ and different $q$-profile, the spheromak has fewer low-order rational surfaces. Especially when sustained, it usually has only one $m=1$ mode unstable at a time, as in the tokamak, so that magnetic turbulence will have a very different character than the RFP. Comparing results in the two devices should thus advance insights into the physics of the magnetic dynamo. 
Like a spheromak, the FRC does not have a center post but has zero toroidal magnetic field and thus zero helicity. The ST is like a spheromak but has a center post providing the primary toroidal field, and it operates with $q>1$. The FRC and ST can be considered as bounding the spheromak with magnetic configurations having zero and strong toroidal fields respectively, and with helicity playing little or no role in the plasma equilibrium and stability.

\section{B. Theory and computations}

Theory and computational support for the spheromak is needed in several areas, to improve our understanding and predictive ability and to interpret data from experiments. Important plasma disciplines which require support include:

- Ideal MHD equilibria. Calculation of axisymmetric MHD equilibria either with a conducting flux conserver or external coils can be done routinely using one of several codes based on the Grad-Shafranov equation. Calculations in the ideal limit can thus be done easily and quickly, for example allowing rapid evaluation of configurations or rapid inversion of experimental data. The models include currents on open magnetic field-lines (surrounding the plasma) and an MHD model of the helicity injector. Little development is needed in this area, except to include rotation if experiments show it to be important.

- Ideal MHD stability. Calculation of stability to low-order, ideal modes in the presence of conducting walls can be carried out using existing codes. Open field-line plasma currents are not included in the codes, and rotation (and shear in the flow) may be important. There is thus a need to extend the computational capabilities for the sustained spheromak. This capability will be essential to evaluate the ideal stability of the sustained spheromak with current down the symmetry axis, as in helicity sustainment experiments.

- Feedback or other control of low-order MHD modes. The calculation of control of the tilt and shift modes on the L/R time scale of the flux conserver is similar to that of the vertical instability in the tokamak, except for the breaking of axisymmetry. The calculation can be done using a combination of equilibrium and stability codes, as was done for the tokamak.

- Resistive MHD. Although there have been some resistive MHD calculations for the spheromak, computational analysis of the threedimensional resistive behavior is still under development. It will be critical for understanding the complex behavior inherent in formation 
and sustainment. Important physics ranges from the startup and sustainment of the spheromak configuration to the linear and nonlinear behavior of current- and pressure-driven modes. These modes play major roles in the related problems of helicity transport into the core plasma and current drive through the dynamo process. Magnetic reconnection often occurs in narrow resistive layers, so a multi-scale approach is needed for accurate modeling. The resulting magnetic fluctuations are expected to dominate energy losses across the equilibrium magnetic flux surfaces, so modeling of the resistive spheromak, including FLR and other "small" effects known to affect the stability, has broad ramifications.

- The microscopic dynamics underlying helicity injection. Helicity injection is a macroscopic concept based on global conservation equations. Understanding the detailed dynamics will lead to the optimization of helicity injection, i.e., maximize creation of toroidal current with minimum degradation of confinement.

- Non-MHD reconnection physics. As spheromaks get hotter, additional, physics (not included in MHD) must be taken into account. In particular, magnetic turbulence will likely be closely related to weakly damped Alfvén modes, and electron inertia and ion kinetic effects may be important at small scales, so magnetic reconnection may be collisionless. The observed behavior of highly collisionless space plasmas suggests that the Taylor principle should persist into the hot, collisionless regime, but the detailed physical mechanisms will probably differ.

- Transport. Transport modeling (energy and particles) and comparison with experiment requires better understanding of the mechanisms in fluctuating and turbulent magnetic fields. Calculations and measurements of the scaling for use in transport codes, as well as firstprinciples simulations, are needed.

- Spheromak edge physics. The spheromak edge is especially important since the spheromak current profile is much flatter than the tokamak. Further, coaxial helicity injection requires that the edge carry substantial discharge current. The physics of the edge, including energy and particle balance, impurity generation and behavior, etc., is critical to extrapolation to high-confinement devices. The role of a divertor will also need study.

- Integrated modeling. Computer science and technology have advanced to the point that integrated modeling including multiple physics issues is becoming feasible. This modeling will correctly include coupling between physics processes on multiple time and spatial scales. Such modeling is probably needed to understand the coupling between the core and edge plasmas, essential to coaxial helicity drive. Other processes, 
such as the current-transport consequences of feedback stabilization of the tilt and shift modes, likely also require integrated modeling.

\section{Applications of spheromak research other than magnetic confinement}

Spheromak plasmas are being used to study plasma science issues including magnetic reconnection (PPPL), the acceleration of charged particles during the reconnection (Swarthmore), and laboratory modeling of solar prominences (Caltech). New results are expected to yield better understanding of these complex processes. Besides contributing to the fundamental infrastructure of scientific knowledge, these basic science expcriments also provide new insights into important issues for confinement experiments.

Spheromaks have other fusion-relevant applications. For example, injection of accelerated spheromak compact tori (CTs) into a tokamak provides an attractive means for fueling a tokamak; injection experiments on a medium sized tokamak (TdeV) have demonstrated the feasibility of this technique. The directed ion energy during injection, $\sim 1 \mathrm{keV}$, is a good match to the rotational energy of the tokamak, thus offering the possibility of momentum injection for control of rotation in advanced tokamaks. The accelerated CT has also been applied to the generation of energetic $x$ rays (Marauder).

\section{Physics Issues and Goals}

The physics of the spheromak has been summarized in the reviews referenced earlier, $[7,8,9,10]$ and in the appendix to this document. The observation of moderate temperatures (several hundred $\mathrm{eV}$ ) in the spheromak, as achieved in a decaying CTX plasma, is important both to demonstrate progress in the fusion application and for carrying out plasma physics experiments in a regime with long mean-free-path and high Lundquist number. To reach these conditions and to proceed to advanced experiments we can define a number of important goals.

\section{A. Scientific issues and goals:}

- Helicity injection and current drive by the magnetic dynamo.

Experiments have demonstrated that helicity is conserved on the energyrelaxation time-scale and lost on the resistive diffusion time, and that the current driven in the spheromak core is determined by the injected helicity, e.g. into the flux conserver from a coaxial plasma gun [14]. Other helicity injection techniques, for example by magnetic flux transfer from a flux core or by a magnetized z-pinch, have also been demonstrated.

Current redistribution by magnetic reconnection has been explicitly 
demonstrated in the spheromak [15]. The coupling between the coaxial helicity injector and the spheromak apparently involves a kink mode on the column of plasma along the symmetry axis of the flux conserver. However, the detailed current-drive mechanisms are not well understood, especially in the sustained dynamo, and may involve processes other than the MHD dynamo. There is need for optimization and pulse-length extension.

Goal: Improve our understanding of helicity, reconnection and the magnetic dynamo in laboratory and space plasmas.

Goal: Understand the mechanism coupling the helicity injector to the spheromak and determine how to sustain the spheromak with minimum perturbation to the axisymmetric configuration.

Goal: Optimize helicity current drive efficiency in the sustained spheromak.

Goal: Develop long pulse and cw helicity injection for the next generations of experiments.

- Energy Confinement. CTX has produced electron temperatures in excess of $100 \mathrm{eV}$ in a decaying plasma, and this result has been confirmed by S-1 and CTCC at Osaka. CTX results were consistent $[12,16]$ with the Rechester-Rosenbluth description of transport in fluctuating magnetic fields, but neither scaling over significant ranges of parameters nor measurements to confirm the detailed physics have been made.

Goal: Achieve $T_{e}$ of several hundred $\mathrm{eV}$ in a sustained spheromak, a value considerably higher than possible with parallel electron thermal conduction being dominant, thus demonstrating good radial energy confinement.

Goal: Study spheromak physics at high electron temperature, both as operation in the fusion regime and to access the long mean-freepath operation necessary to study the physics of resistive tearing and other modes.

Goal: Understand the physics of energy confinement in the presence of the magnetic dynamo, especially during spheromak sustainment.

Goal: Determine the scaling of energy confinement with increasing electron temperature, including the coupled roles of magnetic fluctuations, loses to dissipative processes (e.g. impurity radiation), and other transport mechanisms.

- Beta. The spheromak is generally considered a low-beta device, but experimental electron betas up to $20 \%$ have been achieved in the core [17], well above the expected Mercier limit. Modeling predicts that betapoloidal of several tens of percent is within the Mercier limit if the current profile is properly shaped. Further, calculations indicating verylow growth rates at betas well above the Mercier limit need further 
investigation [18]. Pressure-driven tearing modes are predicted to be unstable, but finite Larmor radius, fluid velocity shear, and other subtle effects may substantially extend the range of stability.

Goal: Understand the beta-limiting processes in the spheromak plasma. Goal: Achieve sufficiently high beta (as measured by beta poloidal) for a fusion reactor, in a system with relevantly high $S\left(S>10^{4}\right)$.

- MHD stability of the core plasma. Deviations of the current profile from the Taylor state are necessary to transport helicity from the plasma edge to the core and to support finite beta. These deviations also provide free energy for MHD instabilities. What is not known is whether magnetic fluctuations saturate at the level necessary to provide the current drive needed to balance resistive losses, or whether the instability mechanisms are robust enough that modes grow until the magnetic field is fully stochastic and confinement is degraded.

Goal: Determine whether current profile control is required to achieve a sufficiently stable plasma for good energy confinement.

Goal: If current profile control is required, develop mechanisms and apply them to the spheromak.

Goal: Evaluate the role of pressure-driven resistive tearing modes in limiting beta.

Goal: Determine stability characteristics and control scenarios (Sec. V.B) for long pulses when resistive wall modes are possible.

- Fueling and particle control. Excessive neutral density in the plasma edge causes energy losses through ionization, radiation and charge exchange. It also makes it very difficult to control the density in the confined plasma, which should operate in the low density, high current density regime presumably required for high temperatures. (Generally, to burn through impurities requires the ratio of current density to plasma density to be in the range $10^{-14}$ to $10^{-13} \mathrm{~A}-\mathrm{m}$.) Minimization of wall recycling is probably necessary to control both hydrogenic densities and impurities, essential for high-performance operation.

Goal: Understand and minimize mass flow from the coaxial source and increase helicity injection efficiency.

Goal: Control neutral density in the edge plasma, reducing recycling by using a divertor, wall conditioning, and other techniques, to minimize the impact on energy confinement and other system parameters.

Goal: Control the impurity content of the sustained spheromak.

- Edge and boundary physics. The spheromak edge plasma carries helicity from the injector to the confined plasma. However, unlike the core, the 
edge has high current densities in a relatively cold plasma, and can interact strongly with the walls (both along field-lines and across to the flux conserver).

Goal: Understand and control the processes which determine the properties of the edge/boundary plasma, including the roles of the current density from helicity injection, atomic and molecular processes, instabilities, impurity generation and other effects due to wall interactions.

- Long-pulse equilibria supported by external coils. For pulse lengths long compared with the $\mathrm{L} / \mathrm{R}$ time of the flux conserver, the plasma hoop stress will be supported by, and shaping determined by, externally applied fields. It is expected, however, that the plasma will require at least a partial flux conserver to reduce the growth rate of the tilt and shift (and other) modes and so reduce the requirements for active feedback control (see below). The transition from an equilibrium supported by the flux conserver to one supported by external coils has not been studied in detail and should be understood.

Goal: Establish equilibria with the hoop stress and spheromak shape controlled by an external magnetic field, and with a flux conserver to reduce MHD growth rates of low-order, ideal modes.

- Control of tilt and shift modes for long pulses by feedback or other mechanisms. Although for short times a flux conserver can eliminate the tilt and shift modes by shaping the plasma, one external mode (at least) is expected to be unstable on the L/R time-scale of the flux conserver. Feedback or other mechanisms will be required for stability for long times.

Goal: If necessary, use feedback control of the $n=1 / m=1$ modes for sustainment long compared with the flux conserver resistive time.

\section{B. Technology issues and goals}

- Long-pulse helicity injection or other current drive for steady-state experiments and reactors. Electrostatic helicity injection is generally an efficient means of sustaining the spheromak. However, the current and power densities in coaxial helicity injection experiments to date are larger than can be sustained without improving the technology or developing new techniques. Opportunities include optimization to reduce the current density on walls and the development of advanced techniques such as extraction of current from a plasma discharge or thermionic emitters. Sustainment by injection of small CTs has been suggested for reactors and might be used in advanced experiments. 
Goal: Develop electrostatic or other helicity injection technology for long-pulse and $\mathrm{cw}$ operation.

- Feedback systems for control of $n=1 / m=1$ mode for times long compared to flux conserver $L / R$ times. Control of the tilt and shift modes in the presence of a resistive wall will likely require feedback, unless sheared rotation or other mechanisms provide sufficient stabilizing force.

Goal: Develop and test feedback systems for the tilt/shift modes (and other low-order modes if necessary), including sensors, feedback circuits, and power systems.

\section{Energy issues and goals}

The most detailed study to date of the spheromak as a reactor was by Hagenson and Krakowski [11]. The study used the CTX geometry and considered a high-power-density reactor based on detailed technology and cost issues as understood at the time. However, the physics assumptions will need revision as understanding improves, and the high power density on the first wall (nearly $20 \mathrm{MW} / \mathrm{m}^{2}$ ) was higher than thought reasonable today. Thus, innovations, such as those described below, are highly desirable to take advantage of the spheromak potential.

- Low density spheromak in the MFE regime. Hagenson and Krakowski showed that a cost-effective reactor is achievable if betas of greater than $10 \%$ can be achieved. However, achieving this goal depends on applying an improved physics understanding and on innovative technology.

Goal: Improve the physics base for the reactor.

- Innovative technologies - Liquid walls. Liquid walls could allow operation at much higher power density than solid walls. Proposals include the use of liquid lithium and FLiBe salt.

Goal: Evaluate the physics of the liquid wall - spheromak plasma interaction to determine whether the concept is viable. Issues include the shielding of the core by the edge plasma and MHD stability in the presence of the liquid.

- Innovative technologies - Wall confined plasma. A pulsed spheromak reactor may be possible. It would be ignited ohmically at low beta, followed by fueling to $\beta \geq 1$ at a rate slower than MHD processes that flatten the pressure gradient, thus utilizing wall confinement. The configuration would continuously relax the magnetic field to a stable Taylor state, fast enough to overpower edge cooling and core heat losses. 
The wall would be liquid or other high-power density material, and the device would operate with a pulse length $\approx$ the $L / R$ time.

Goal: Evaluate critical physics issues such as the power thresholds to sustain a transient, high-beta detached state during the wallconfinement phase, and the magnetic and electrostatic turbulence at $\beta \geq 1$.

- Innovative technologies - Magnetized Target Fusion. Magnetized Target Fusion is proposed to operate at very high plasma betas. The spheromak is a candidate for the MTF core [19], although very high densities $\left(10^{23}\right.$ $10^{24} \mathrm{~m}^{-3}$ ) are required.

Goal: As the MTF approach is explored further, review its needs to determine whether the spheromak offers a credible and promising core plasma for the concept.

\section{Existing Spheromak Programs and Resources}

\section{A. Confinement and helicity injection experiments}

SSPX: The Sustained Spheromak Physics Experiment (SSPX) [16], under construction at LLNL, will contribute to all the scientific goals except those requiring long pulses. Preliminary experiments are also planned to examine the initial phases of the transition from the short pulse, wall supported regime to the long pulse regime, thus laying the physics base for a proof-ofprinciple experiment. Specific goals include:

- Demonstrate that electron and ion temperatures of a few hundred electron volts can be achieved in a steady-state spheromak plasma sustained by a magnetic dynamo ("helicity injection").

- Relate energy confinement quantitatively to the magnetic turbulence accompanying the dynamo and use this knowledge to optimize performance.

- Measure the magnetic field profiles and magnetic turbulence in the plasma and relate these to the science of the magnetic dynamo which drives the current in the plasma. Understanding these phenomena includes studies of resistive MHD in the plasma.

- Examine experimentally the beta limit of the sustained spheromak.

- Understand the initial phases of the transition of the plasma from an equilibrium supported by a magnetic-flux conserving wall to one supported by external coils.

The plasma will be formed in a $1 \mathrm{~m}$ diameter flux conserver, with the plasma initiated by a $10 \mathrm{kV}, 0.5 \mathrm{MJ}$ startup capacitor bank. Sustainment will 
be provided by a $5 \mathrm{kV}, 1.5 \mathrm{MJ}$ pulse-forming network, providing a nominal pulse length $\geq 2 \mathrm{~ms}$. The separation of the two power systems will allow simultaneous optimization of startup and sustainment currents. Copper walls will be protected by a tungsten-sprayed coating; baking, discharge cleaning and boronization will be used to create clean conditions. The flux conserver incorporates a divertor for particle and impurity control. The experimental design is thus directed to minimizing energy losses due to impurities and to provide means of density control, essential to achieving the temperatures needed to address physics goals involving MHD stability and related transport.

Experiments to explore and optimize the spheromak magnetic geometry will use a bias magnetic flux provided by external coils. The resulting configurational flexibility includes the option of extending the outside scrapeoff layer into the divertor to enhance particle and impurity control. A Taylor helicity drive configuration, with current passing from the injector cathode on the top of the plasma into the divertor on the bottom can also be tested; this may provide a less disruptive current drive than the conventional geometry which has current returning around the outside of the plasma but with the location of the wall return not well controlled.

Planned diagnostics include an ultra-short-pulsed reflectrometer, developed with the collaboration of UC Davis, operating in both the O- and Xmodes to measure density and magnetic field profiles. Coupling between the two modes is predicted to measure the shear in the magnetic field, and thus to be sensitive to the magnetic fluctuations in the plasma.

Improving our understanding of the physics issues (Section V) will require measurement of profiles, e.g. of density, temperature, and current, and relating these to magnetic fluctuations as measured by edge magnetic probes and the reflectrometer. Although probes can be inserted into the edge plasma, a high Lundquist number, $S$, cannot be maintained if they are inserted into the core, so reliance on remote measurements is required. Evaluating energy confinement and transport in the core also requires knowledge of profiles as demonstrated by tokamak experiments. The experimental plan for SSPX includes such measurements as diagnostics become available on the facility.

Caltech Helicity Experiment: The Caltech group is investigating the detailed physics underlying helicity injection. A magnetized coaxial plasma gun is fircd into a large ( $1.3 \mathrm{~m}$ diameter, $2 \mathrm{~m}$ long) vacuum chamber and the evolution of the magnetic topology is followed on the sub-microsecond timescale using high speed photography and magnetic probes. These photos show the development of spiraling magnetic flux tubes that emanate from the center electrode, progressively bulge out, and then return to the outer electrode. The photos show that even though the gun is axisymmetric, the 
configuration develops reproducible non-axisymmetric discrete flux tubes. The sense of the spirals reverses when the bias magnetic field is reversed. A stereographic camera system is now being constructed which will provide $3 \mathrm{D}$ movies of the helicity injection process.

SSX: The Swarthmore Spheromak Experiment (SSX) at Swarthmore College has been operational since 1996. The SSX group has performed scaling studies of the formation and equilibrium of spheromaks, and examined detailed physics in decaying plasmas [20]. SSX spheromaks are formed with a magnetized coaxial plasma gun and equilibrium is established in both small $\left(\mathrm{d}_{\text {small }}=0.16 \mathrm{~m}\right)$ and large $\left(\mathrm{d}_{\text {large }}=3 \mathrm{~d}_{\text {small }}=0.50 \mathrm{~m}\right)$ copper flux conservers. Using magnetic probe arrays they have verified that spheromak formation is governed solely by gun physics (in particular the ratio of gun current to flux, $\left.\mu_{0} I_{\text {gun }} / \Phi_{\text {gun }}\right)$ and is independent of the flux-conserver dimensions. They have also verified that equilibrium in this cold plasma is well described by the force-free condition at all scales. Current work at SSX involves measuring correlated magnetic reconnection and energetic ion flow events [21]. The reconnection layer is measured with probe arrays and ion flow is directly measured with a retarding grid energy analyzer.

\section{B. Physics of reconnection}

MRX: The MRX device at PPPL is fully devoted to study of the fundamental physics of magnetic reconnection, which is an essential element for fusion research as well as other plasma physics branches such as space physics [22]. Recently the SPIRIT device has been proposed by the MRX group for compact toroid research. These experiments continue work originated with merging spheromaks on TS-3 at the University of Tokyo, which have demonstrated that when the helicities are of the same sign, merging results in a new spheromak, but when they are opposed (and approximately equal), they result in an FRC.

Reconnection experiments will be also carried out extensively on SSX at Swarthmore College.

\section{CT injection for fueling}

The acceleration of spheromaks with uses that include fueling tokamaks, was initiated on the RACE experiment at LLNL. Subsequent work in the US was carried out on the Marauder experiment at Air Force Phillips Lab (but not for fueling), at Caltech, and on the DDT experiment by UC Davis (located at LLNL). Only the latter experiment is currently active. In Japan, the Himeji Institute of Technology (HIT) is presently installing a spheromak injector on the JFT-2M tokamak, and the concept is being studied for possible application to the Large Helical Device and JT-60. In Canada, CCFM has completed 
fueling studies on TdeV. Work continues at the University of Saskatchewan with studies of CT injection with variation of the injector angle.

\section{Diagnostic development}

As the spheromak program proceeds, it will be necessary to apply many of the sophisticated diagnostics developed by the magnetic fusion program, typically directed to tokamak development. New diagnostics will also be needed to address issues of particular importance to plasmas based on helicity drive.

The use of probes for multiple measurements simultaneously has been productive and should be extended. Examples include the use of the " $\lambda$ probe" to measure local currents and magnetic fields on SPHEX and measurements of magnetic fluctuations and heat/particle flows on the MST RFP. An ultra-short pulse reflectrometer is being developed for use on SSPX in collaboration with UC Davis. By operating with both O- and X-modes, the density and magnetic field profiles can be determined. The coupling between the two modes is determined by the magnetic shear, and is thus particularly sensitive to magnetic tearing modes. Support will undoubtedly be needed for other new diagnostics as well; for example, a diagnostic neutral beam has significant potential for determining profiles of plasma parameters and fluctuations.

\section{Collaborations and Contributors to the White Paper}

Collaborations among the spheromak community and with other parts of the MFE community will apply skills, experience, and equipment to advancing the spheromak. Several of these already in place include applying physics and diagnostics to SSPX by the University of Washington and by the University of California at Berkeley and at Davis. General Atomics undertook the MHD stability analysis of SSPX and plans further participation in the experiment. Informal collaborations include Swarthmore, PPPL, Caltech, and LANL. Proposals have been made for funding to support formal collaborations on SSPX by LANL and PPPL. Discussions with the RFP community, especially at the University of Wisconsin, could bring their experience and edge diagnostics to SSPX. Swarthmore and PPPL are collaborating on reconnection experiments using colliding spheromaks.

Although there are presently no spheromak experiments outside the US, there is considerable interest, especially in Japan. The program needs to encourage participation there and elsewhere about the world so as to broaden the base of physics and analysis on spheromaks. 
This white paper was prepared by a broad representation from the spheromak community. Contributors include E. B. Hooper, editor (LLNL), C. W. Barnes (LANL), P. M. Bellan (Caltech), M. R. Brown (Swarthmore), J. C. Fernández (LANL), T. K. Fowler (U. California at Berkeley), D. N. Hill (LLNL), T. R. Jarboe (U. Washington), L. L. LoDestro (LLNL), H. S. McLean (U. California at Davis), M. J. Schaffer (General Atomics), K. I. Thomassen (LLNL), and M. Yamada (PPPL).

\section{References (main text)}

1. L. Woltjer, "A Theorem on Force-Free Magnetic Fields," Proc. National Acad. Sci. (US), 44, 489 (1958).

2. E. E. Nolting, P. E. Jindra, and D. R. Wells, "Experimental study of forcefree collinear plasma structures," J. Plasma Phys. 9, 1 (1973).

3. J. B. Taylor, "Relaxation of Toroidal Plasma and Generation of Reverse Magnetic Fields," Phys. Rev. Letters 33, 1139 (1974); "Relaxation and magnetic reconnection in plasmas," Rev. Mod. Phys. 58, 741 (1986).

4. M. N. Rosenbluth and M. N. Bussac, "MHD stability of spheromak," Nucl. Fusion 19, 489 (1979).

5. T. R. Jarboe, F. J. Wysocki, J. C. Fernández, I. Henins, and G. J. Marklin, "Progress with energy confinement time in the CTX spheromak," Phys. Fluids B 2, 1342 (1990).

6. F. J. Wysocki, J. C. Fernández, T. R. Jarboe and G. J. Marklin, "Evidence for a Pressure-Driven Instability in the CTX Spheromak," Phys. Rev. Lett. 61, 2457 (1988).

7. M. Yamada, "Review of experimental spheromak research and future prospects," Fusion Techn. 9, 38 (1986).

8. B. L. Wright, "Field reversed configurations and spheromaks," Nucl. Fusion 30, 1739 (1990).

9. J. C. Fernández, R. E. Chrien, F. J. Wysocki, R. M. Mayo, and I. Henins, “A view on advances in spheromak understanding and parameters," in $S$. Ortolani and E. Sindoni (Eds.), Physics of Alternate Magnetic Confinement Systems, (SiF, Bologna, 1991), p. 191.

10. T. R. Jarboe, "Review of spheromak research," Plasma Phys. Contr. Fusion 36, 945 (1994).

11. R. L. Hagenson and R. A. Krakowski, Fusion Techn. 8, 1606 (1985).

12. T. K. Fowler, "Theoretical Aspects of Energy Confinement in Spheromaks," Fusion Techn. 29, 206, (1996).

13. K. Katayama and M. Katsurai, "Three-dimensional numerical simulations of the relaxation process in spheromak plasmas," Phys. Fluids 29, 1939 (1986). 
14. C. W. Barnes, J. C. Fernández, I. Henins, H. W. Hoida, T. R. Jarboe, S. O. Knox, G. J. Marklin, and D. F. McKenna, "Experimental determination of the conservation of magnetic helicity from the balance between source and spheromak," Phys. Fluids 29, 3415 (1986).

15. Y. Ono, M. Yamada, A. C. Janos, and F. M. Levinton, "Experimental study of the relaxation cycle of a decaying spheromak in an external magnetic field," Phys. Fluids B 3, 1452 (1991).

16. E. B. Hooper, J. H. Hammer, C. W. Barnes, J. C. Fernández, amd F. J. Wysocki, "A reexamination of spheromak experiments and opportunities," Fusion Techn. 29, 191 (1996).

17. F. J. Wysocki, J. C. Fernández, I. Henins, T. R. Jarboe, and G. J. Marklin, "Evidence for a Pressure-Driven Instability in the CTX Spheromak," Phys. Rev. Letters 61, 2457 (1988).

18. P. Gautier, R. Gruber, and F. Troyon, "Numerical Study of the Ideal-MHD Stability Limits in Oblate Spheromaks," Nucl. Fusion 21, 1299 (1981).

19. Magneto-Inertial Fusion - Research and Development Needs, March 1998.

20. C. G. R. Geddes, T. W. Kornack, and M. R. Brown, "Scaling studies of spheromak formation and equilibrium," Phys. Plasmas 5, 1027 (1998).

21. T. W. Kornack, P. K. Sollins, and M. R. Brown, "Experimental observation of correlated magnetic reconnection and Alfvénic ion jets," submitted to Phys. Rev. E, Rapid Communications (1998).

22. M. Yamada, H. Ji, S. Hsu, T. Carter, R. Kulsrud, N. Bretz, F. Jobes, Y. Ono, and F. Perkins, "Study of driven magnetic reconnection in a laboratory plasma," Phys. Plasmas 4, 1936 (1997). 


\section{Appendix: Physics Issues and Status}

\section{Appendix 1. Spheromak physics}

Helicity injection, reconnection, and current drive. A spheromak in the limiting case of zero beta is an example of a "force-free" magnetofluid configuration with $\mathbf{J} \times \mathbf{B}=0$. (The concept can be extended to include currents associated with finite beta.) The magnetic field in the force-free limit is given by $\nabla \times \mathbf{B}=\lambda \mathbf{B}$. A magnetofluid evolves into a nearly force-free state through processes involving "relaxation." Turbulence, allied with small resistivity, allows the plasma rapid access to a minimum-energy state at constant global helicity in a time short compared with the usual resistive diffusion time.

The topological invariant called magnetic helicity (defined $K \equiv \int \mathbf{A} \bullet \mathbf{B} d^{3} x$ ) plays a key role in spheromak physics. Since magnetic flux can be expressed $\Phi \equiv \int \mathbf{B} \bullet \mathbf{d}^{2} \mathbf{x}$ or $\Phi \equiv \int \mathbf{A} \bullet \mathbf{d}^{1} \mathbf{x}$ (where the integrals extend over a suitable boundary), the magnetic helicity is the product of linked poloidal and toroidal flux elements, $K=\iint d \Phi_{1} d \Phi_{2}$ [1]. It can be shown [2,3] that if the magnetic energy of a zero-beta spheromak $\left[W \equiv \int\left(B^{2} / 2 \mu_{0}\right) d^{3} x\right]$ is minimized subject to the constraint of constant global helicity, the resulting equilibria satisfy the condition $\nabla \times \mathbf{B}=\lambda \mathbf{B}$. The quantity $\lambda$ (the Lagrange multiplier in the calculation) is a constant eigenvalue with units of (length) ${ }^{-1} . \lambda$ is a measure of the ratio of current density to magnetic field, $\mu_{0} J / B$, in the plasma as well as the ratio of energy to helicity, $2 \mu_{0} W / K$. More generally, $\lambda$ is a function of flux, with its average related to an eigenvalue of the geometry; at finite beta perpendicular currents flow to support the pressure gradient.

The rate at which helicity is injected into a system is given by $\dot{K}=2 \dot{\Phi}_{1} \Phi_{2}$. In the case of a spheromak formed by a coaxial magnetized plasma gun, $\dot{\Phi}_{1}=\dot{\Phi}_{\text {tor }}=V_{\text {gun }}$ and $\Phi_{2}=\Phi_{\text {pol }}=\Phi_{g u n}$. Helicity balance between gun and spheromak has been verified in detail by the CTX group [4]. Steady-state spheromak sustainment (current drive) becomes tantamount to applying a sufficient voltage across the gun terminals while maintaining the linked gun flux.

The issue of magnetic reconnection enters spheromak physics at several levels. First, during the formation process, the gun flux $\Phi_{\text {gun }}$ must expand away from the gun by magnetic reconnection in order to form $\Phi_{p o l}$ of the spheromak. In a similar way, spheromak sustainment requires continuous (perhaps bursty) reconnection events as the poloidal flux is replenished. Finally, any relaxation process (from a change in boundary conditions, for example) requires dissipation through magnetic reconnection as the spheromak minimizes energy subject to the constraint of constant helicity. 
Edge and boundary physics. Just as helicity can be injected into a spheromak by applying a voltage across linked flux so can helicity be removed from a spheromak. In particular, if there is significant edge flux that passes through regions of neutral gas, then helicity can be dissipated at a rate $\dot{K}=2 V_{\text {edge }} \Phi_{\text {edge }}[5]$. The CTX group determined that $V_{\text {edge }} \approx \int \mathrm{E}_{\text {Paschen }} \bullet \mathbf{d x}$, where $\mathbf{E}_{\text {Paschen }}$ is the Paschen electric field required to ionize the neutral gas. It is for this reason that minimal edge error flux and neutral density control are critical for fusion grade spheromaks. Indeed, the highest performance CTX spheromaks were formed in close fitting, Ti gettered solid copper flux conservers $[6,7]$.

Long pulse helicity injection or other current drive. Experimental work on spheromaks was begun in the early 1980's with the CTX program at Los Alamos [8] and the S-1 program at Princeton [9], among others. The goal of this early work was to explore the spheromak as a magnetic confinement fusion configuration. Although spheromak formation proved to be relatively straightforward, researchers found that these plasmas were plagued by low temperatures, short confinement times and instabilities (notably the tilt instability $[10,11])$. In addition, the conversion of energy stored in capacitor banks to energy stored in the spheromak was found to be highly inefficient in early spheromak experiments.

By the late 1980's, the CTX group had produced stable, 3-tesla spheromaks with electron temperatures in excess of $400 \mathrm{eV}$ (during decay) and decay times of several ms [12]. This success was due in part to the use of close fitting, solid flux conservers and proper wall conditioning $[13,14,15,10]$. The key to proper conditioning was the incorporation of titanium gettering of the flux conserver walls $[16,5]$. In addition, the CTX group had perfected the slowformation technique [17] so that spheromaks could be sustained for times long compared to their resistive decay times with reasonably efficient use of capacitor-bank energy.

The CIX group developed a magnetized, coaxial plasma gun to form and sustain spheromaks. The gun consists of an inner electrode which is magnetized by an external coil. Magnetic flux $\Phi_{\text {gun }}$ links the inner to the outer clectrode. Gas is puffed into the annular gap and high voltage is applied. The high voltage breaks down the gas and current flows from the inner to the outer electrode generating toroidal magnetic flux which encircles the inner electrode. At sufficiently high current, the toroidal flux entrained in the magnetofluid distends the gun flux and a spheromak is formed with $\Phi_{\text {gun }}$ becoming the poloidal flux. The voltage that appears between the inner and outer electrode is determined by the rate at which toroidal flux is ejected from the gun, $V_{\text {gun }} \approx d \Phi_{\text {gun }} / d t$ [18]. During formation, helicity is injected at a rate $2 V_{g u n} \Phi_{g u m}$. As the plasma relaxes to the equilibrium state, some of the injected toroidal flux twists with respect to the magnetofluid and reconnects with the poloidal flux. The impedance and efficiency of the coaxial plasma 
gun are well understood and efficient guns have been experimentally demonstrated [18].

Spheromaks can be sustained near the force-free state by continually injecting helicity (and energy) at a rate that balances helicity (and energy) dissipation. The efficiency of this process depends on the relative sizes of the spheromak and helicity injector. The SSPX experiment will address the issue of sustainment directly. It's also possible to use a smaller gun for spheromak formation and a separate large, efficient gun for sustainment [19]. It has been shown that energy coupling efficiency approaches unity if the gun and spheromak are of comparable size. A large gun should be able to operate at lower current and therefore lower voltage. In addition, a gun matched to the size of the spheromak is expected to cause less perturbation to the equilibrium.

Relation to other areas of plasma physics (e.g. space physics, reconnection). A number of researchers have identified solar flares as force-free magnetic structures with properties similar to those of spheromaks [20,21]. Heyvaerts and Priest [22] invoked the Taylor hypothesis to explain the relaxation of solar flares and subsequent heating of the surrounding corona. Kusano, et al. [23] performed 3D simulations to demonstrate the spontaneous generation of the "Taylor-Heyvaerts-Priest" state in the solar corona. Vekstein and coworkers have studied the dynamics of force-free coronal equilibrium structures (one half of a very large spheromak) slowly perturbed at the footpoints by photospheric motion $[24,25,26,27]$. There is also considerable evidence that flare-associated reconnection is responsible for energetic particles in the corona. Astrophysical research also suggests that reconnection structures are fully three dimensional $[28,29,30,31,32]$. Once reconnection occurs, energy is converted to $x$-rays [33], energetic electrons [34], plasma jets [35], and heated plasma [36,37]. Magnetic reconnection has also been inferred in solar flares using high resolution, time-resolved $\mathrm{H}_{\text {alpha }}$ spectrography [38] as well as radio frequency measurements [39].

There is abundant experimental, theoretical and computational evidence that certain constrained turbulent fluid systems self-organize into large scale structures. Examples include two dimensional (geostrophic) fluids, guiding center plasmas, pure electron plasmas, as well as two and three dimensional magnetofluids such as reversed-field pinches and spheromaks. The theoretical understanding of relaxation phenomena is divided into two quite different constructs: selective decay (of which the minimal energy subject to constant helicity theory is a subset) and maximal entropy [40]. Experimental evidence from both 2D fluids and from 3D magnetofluids is consistent with the selective decay hypothesis. Spheromak and RFP relaxation are the major examples of self-organization in 3D magnetofluids. However, high resolution computational evidence strongly suggests that formation of large scale structures is dictated by maximal entropy principles rather than selective decay. 


\section{Appendix 2. Recent results from the SPHEX experiment}

The SPHEX spheromak at UMIST in Manchester, England operated from about 1989 to 1997, so many results are not in the review articles. The SPHEX spheromak was gun-produced (similar to CTX) and sustained in a $1 \mathrm{~m}$ diameter solid copper flux conserver designed with rounded contours to minimize edge error flux. The main issues addressed by the SPHEX group were (1) division of the plasma into a high electric field central column and low electric field toroidal annulus, (2) study of the global $n=1$ mode responsible for carrying energy (and helicity) from the column to the annulus and (3) the MHD dynamo responsible for current drive in the annulus. In addition, the SPHEX group has emphasized that the spheromak plasma is, in general, "partially relaxed" (i.e. not in a constant $\lambda$ state) particularly during sustainment [41].

Analysis of Langmuir and magnetic probe measurements showed that the SPHEX spheromak (and perhaps all gun-sustained spheromaks) had a high clectric field central column $(600 \mathrm{~V} / \mathrm{m})$ and a low electric field annulus [42]. Most of the current from the inner electrode of the gun was found to return through the copper flux conserver $(90 \%)$ rather than through plasma $(10 \%)$ thereby producing a net toroidal field at the wall. A large amplitude, coherent $\mathrm{n}=1$ mode (also observed on CTX) was shown to be responsible for energy transport from the column to the annulus. The Poynting flux associated with this oscillation was about $10 \mathrm{MW} / \mathrm{m}^{2}$.

Experiments were performed on a spheromak with a current-carrying central rod (a rodomak) [43]. It was found that a pre-existing toroidal field improves energy coupling from the gun to the plasma. This experiment was an important precursor to the HIT experiments at the University of Washington.

Careful measurements were performed of the current density and $\lambda$ profiles in SPHEX using a novel Rogowski/magnetic probe [44]. They found a discontinuity in the profiles at the interface between the column and the annulus. $J(r)$ and $\lambda(r)$ in the annulus are consistent with the linear $\lambda(r)$ profiles found in CTX.

Correlations were measured between fluctuations of $\mathbf{E}$ and $\mathbf{B}$ to study the MHD dynamo on SPHEX [45]. A dynamo electric field was constructed as $E_{d y n}=|\tilde{\mathbf{v}} \times \tilde{\mathbf{B}}| \approx \tilde{\mathbf{E}}_{\perp} \bullet \tilde{\mathbf{B}}_{\perp} / B$ which can drive toroidal current in a relaxed spheromak (even though $E_{e x t}=0$ ). They showed that $E_{d y n}$ was sufficient to drive the toroidal current on the magnetic axis, though at the edge of the annulus $E_{d y n}$ actually reverses sign to give an "anti-dynamo" which extracts helicity from the plasma column along the geometric axis. 
Summaries of the SPHEX project have recently appeared emphasizing the "rodomak" configuration [46], ion energy measurements [47], general SPHEX operation [48], the structure of the $n=1$ mode [49], and the effects of instabilities in the plasma [50].

\section{References (Appendix)}

1. H. K. Moffatt, "Magnetic Field Generation in Electrically Conducting Fluids," Cambridge University Press, New York (1978).

2. J. B. Taylor, "Relaxation of Toroidal Plasma and Generation of Reverse Magnetic Fields," Phys. Rev. Lett. 33, 1139 (1974).

3 . J. B. Taylor, "Relaxation and Magnetic Reconnection in Plasmas," Rev. Mod. Phys. 58, 741 (1986).

4. C. W. Barnes, J. C. Fernández, I. Henins, H. W. Hoida, T. R. Jarboe, S. O. Knox, G. J. Marklin and K. F. McKenna, "Experimental Determination of the Conservation of Magnetic Helicity From the Balance Between Source and Spheromak," Phys. Fluids 29, 3415 (1986).

5. J. C. Fernández, Cris W. Barnes, T. R. Jarboe, I. Henins, H. W. Hoida, P. Klingner, S. O. Knox, G. J. Marklin and B. L. Wright, "Energy Confinement Studies in Spheromaks with Mesh Flux Conservers," Nucl. Fusion 28, 1555 (1988).

6. F. J. Wysocki, J. C. Fernández, I. Henins, T. R. Jarboe and G. J. Marklin, "Improved Energy Confinement in Spheromaks with Reduced Field Errors," Phys. Rev. Lett. 65, 40 (1990).

7. T. R. Jarboe, F. J. Wysocki, J. C. Fernández, I. Henins and G. J. Marklin, "Progress with energy confinement time in the CTX spheromak," Phys. Fluids B 2, 1342, (1990).

8. T. R. Jarboe, et al., "Motion of a Compact Toroid inside a Cylindrical Flux Conserver," Phys. Rev. Lett. 45, 1264 (1980).

9. M. Yamada, et al., "Quasistatic Formation of the Spheromak Plasma Configuration," Phys. Rev. Lett. 46, 188 (1981).

10. A. Bondeson, G. Marklin, Z. G. An, H. H. Chen, Y. C. Lee and C. S. Liu, "Tilting Instability of a Cylindrical Spheromak," Phys. Fluids 24, 1682 (1981).

11. J. M. Finn and W. M. Manheimer and E. Ott, "Spheromak Tilting Instability in Cylindrical Geometry," Phys. Fluids 24, 1336 (1981).

12. T. R. Jarboe, "Review of spheromak research," Plasma Phys. and Controlled Fusion 36, 945 (1994)

13. T. R. Jarboc, et al., "The Ohmic Heating of a Spheromak to $100 \mathrm{eV}$," Phys. Fluids 27, 13 (1984).

14. C. W. Barnes, et al., "Spheromak Formation and Operation with Background Filling Gas and a Solid Flux Conserver in CTX", Nucl. Fusion 24, 267 (1984). 
15. C. W. Barnes, et al., "Zero-Dimensional Energy Balance Modelling of the CTX Spheromak Experiment," Nucl. Fusion 25, 1657 (1985).

16. F. J. Wysocki, J. C. Fernández, T. R. Jarboe and G. J. Marklin, "Evidence for a Pressure-Driven Instability in the CTX Spheromak," Phys. Rev. Lett. 61, 2457 (1988).

17. T. R. Jarboe, et al., "Slow Formation and Sustainment of Spheromaks by a Coaxial Magnetized Plasma Source," Phys. Rev. Lett. 51, 39 (1983).

18. C. W. Barnes, T. R. Jarboe, G. J. Marklin, S. O. Knox and I. Henins, "The Impedance and Energy Efficiency of a Coaxial Magnetized Plasma Source used for Spheromak Formation and Sustainment," Phys. Fluids B 8, 1871 (1990).

19. M. R. Brown and A. Martin, "Spheromak Experiment using Separate Guns for Formation and Sustainment," Fusion Tech. 30, 300 (1996).

20. T. Gold and F. Hoyle, Mon. Not. Royal Astron. Soc. 120, 89 (1960).

21. C. W. Barnes and P. A. Sturrock, "Force-free Magnetic Field Structures and their role in Solar Activity," Ap. J. 174, 659 (1972).

22. J. Heyvaerts and E. R. Priest, "Coronal Heating by Reconnection in dc Current Systems. A Theory based on Taylor's Hypothesis," Astron. Astrophys. 137, 63 (1984).

23. K. Kusano, et al., "Three-dimensional Simulation Study of the Magnetohydrodynamic Relaxation Process in the Solar Corona. I. Spontaneous Generation of the Taylor-Heyvaerts-Priest State," Ap. J. 433, 361 (1994).

24. G. E. Vekstein, E. R. Priest and T. Amari, "Formation of Current Sheets in Force-free Magnetic Fields," Astron. Astrophys. 243, 492 (1991).

25. G. E. Vekstein, E. R. Pricst and C. D. C. Steele, "On the Problem of Magnetic Coronal Heating by Turbulent Relaxation," Ap. J. 417, 781 (1993).

26. G. E. Vekstein, E. R. Priest and R. Wolfson, "Coronal Magnetic Field Evolution under Reconnective Relaxation," Space Science Reviews 70, 303 (1994).

27. R. Wolfson, G. E. Vekstein and E. R. Priest, "Non-linear Evolution of the Coronal Magnetic Field under Reconnective Relaxation," Ap. J. 428, 345 (1994).

28. D. W. Longcope and H. Strauss, "Spontaneous Reconnection in Line-tied Flux Tubes," Ap. J. 426, 742 (1994).

29. D. W. Longcope and R. N. Sudan, "Evolution and Statistics of Current Sheets in Coronal Magnetic Loops," Ap. J. 437, 491 (1994).

30. D. W. Longcope and H. R. Strauss, "The Form of Ideal Current Layers in Line-tied Magnetic Fields," Ap. J. 437, 851 (1994).

31. D. W. Longcope and S. C. Cowley, "Current Sheet Formation along Threedimensional Magnetic Separators," Phys. Plasmas 3, 2885 (1996).

32. I. J. D. Craig, et al., "An Exact Solution for Steady State Magnetic Reconnection in Three Dimensions," Ap. J. 455, L197 (1995). 
33. S. Masuda, T. Kosugi, H. Hara, S. Tsuneta and Y. Ogawara, "A Loop-top Hard X-ray Source in a Compact Solar Flare as Evidence for Magnetic Reconnection," Nature 371, 495 (1994).

34. D. J. Forrest and E. J. Chupp, "Simultaneous acceleration of electrons and ions in solar flares," Nature 305, 291 (1983).

35. K. Shibata, et al., "Hot-plasma Ejections Associated with Compact-loop Solar Flares," Ap. J. 451, L83 (1995).

36. S. Tsuneta, "Structure and Dynamics of Magnetic Reconnection in a Solar Flare," Ap. J. 456, 840 (1996).

37. S. Tsuneta, "Interacting Active Regions in the Solar Corona," Ap. J. 456, L63 (1996).

38. P. Demoulin, et al., "Evidence for Magnetic Reconnection in Solar Flares," Astron. Astrophys. 271, 292 (1993).

39. P. K. Manoharan, "Evidence for Large Scale Solar Magnetic Reconnection from Radio and X-ray Measurements," Ap. J. 468, L73 (1996).

40. M. R. Brown, "Experimental Evidence of Rapid Relaxation to Large-Scale Structures in Turbulent Fluids: Selective Decay and Maximal Entropy," J. Plasma Physics 57, 203 (1997).

41. D. A. Kitson and P. K. Browning, "Partially Relaxed Magnetic Field Equilibria in a Gun-Injected Spheromak," Plasma Phys. and Controlled Fusion 32, 1265 (1990).

42. P. K. Browning, et al., "Power Flow in a Gun-Injected Spheromak Plasma," Phys. Rev. Lett. 68, 1718 (1992).

43. P. K. Browning, et al., "Injection and Sustainment of Plasma in a Preexisting Toroidal Field using a Coaxial Helicity Source," Phys. Rev. Lett. 68, 1722 (1992).

44. R. Martin, S. J. Gee, P. K. Browning and M. G. Rusbridge, "The Direct Determination of the Current Density and $\mu$ Profiles of a Spheromak," Plasma Phys. and Cont. Fusion 35, 269 (1993).

45. A. al-Karkhy, et al., "Observations of the Magnetohydrodynamic Dynamo Effect in a Spheromak Plasma," Phys. Rev. Lett. 70, 1814 (1993).

46. P. K. Browning, J. R. Clegg, R. C. Duck and M. G. Rusbridge, "Relaxed and partially relaxed magnetic equilibria in tight-aspect-ratio tori," Plasma Phys. and Controlled Fusion 35, 1563 (1993).

47. K. J. Gibson, S. J. Gee and M. G. Rusbridge, "Ion Energy Measurements in the SPHEX Spheromak," Plasma Phys. and Controlled Fusion 37, 31 (1995).

48. M. G. Rusbridge, et al. "The Design and Operation of the SPHEX Spheromak," Plasma Phys. and Controlled Fusion 39, 683 (1997).

49. R. C. Duck, et al. "Structure of the $n=1$ Mode responsible for Relaxation and Current Drive during Sustainment of the SPHEX Spheromak," Plasma Phys. and Controlled Fusion 39, 715 (1997).

50. G. Cunningham, "Impurities and impurity transport in the spheromak SSPX," Plasma Phys. Control. Fusion, 39, 1339 (1997). 


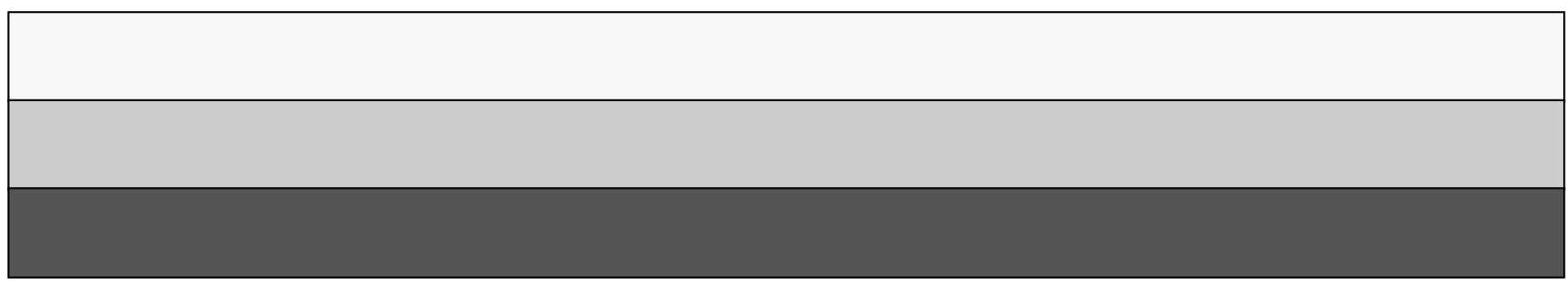

